\title{
PROTÓTIPO PARA A CONSTRUÇÃO DE UM OBJETO DE APRENDIZAGEM DO CONCEITO DE NÚMERO PARA CRIANÇAS COM SÍNDROME DE DOWN
}

Janiele de Souza Santos, Gunnar Correa Pereira Ferreira, Manoel Osmar Seabra Júnior

\begin{abstract}
Universidade Estadual Paulista - Júlio de Mesquita Filho, Programa de Pós-Graduação em Educação, Presidente
\end{abstract} Prudente, São Paulo. E-mail: janisssantos16@gmail.com

\section{RESUMO}

Estudantes com Síndrome Down apresentam dificuldades relacionadas aos conteúdos Matemáticos, principalmente à construção do conceito de número. Assim, na intenção de implementar uma possibilidade de aprendizagem em circunstância lúdica a estes estudantes, foi proposto a construção de um objeto de aprendizagem, como recurso pedagógico para auxiliar o professor no processo de ensino e aprendizagem matemática. Portanto, o objetivo deste trabalho é descrever a construção metodológica do protótipo de um objeto de aprendizagem que estimule os sete processos mentais básicos para a construção do conceito de número. A metodologia pode ser considerada descritivo-analítica e, a construção do objeto de aprendizagem é baseada nos estudos de Amante e Morgado. Os resultados são demonstrados pela metodologia de elaboração do protótipo para trabalhar cada um dos sete processos para a construção do conceito de número. Para projeções futuras, considera-se importante a construção do software, bem como a avaliação da usabilidade do mesmo.

Palavras-chave: Educação Especial. Síndrome de Down. Objeto de Aprendizagem. Conceito de número.

\section{PROTOTYPES FOR A CONSTRUCTION OF A NUMBER CONCEPT LEARNING OBJECTIVE FOR CHILDREN WITH DOWN SYNDROME}

\begin{abstract}
Students with Down Syndrome present difficulties related to Mathematical contents, mainly to the construction of the number concept. Thus, in the intention to implement a learning possibility in playful circumstances to these students, it was proposed the construction of a learning object, as a pedagogical resource to assist the teacher in the process of teaching and mathematical learning. Therefore, the objective of this work is to describe the methodological construction of the prototype of a learning object that stimulates the seven basic mental processes for the construction of the concept of number. The methodology can be considered descriptive-analytical and, the construction of the learning object is based on the studies of Lover and Morgado. The results are demonstrated by the methodology of elaboration of the prototype to work each one of the seven processes for the construction of the number concept. For future projections, it is considered important the construction of the software, as well as the evaluation of the usability of the same.
\end{abstract}

Keywords: Special Education. Down's syndrome. Learning Object. Concept of number. 


\section{INTRODUÇÃO}

O conceito de número e o processo de construção do conhecimento têm sido assuntos de interesse para a pesquisa em diversos campos, tanto da área Educacional, como é o caso dos estudos de Dewey (1898), Piaget (1952), Kamii (1990), Vergnaud (1993); quanto na área da Psicologia, com os estudos de Vygotsky (1983), Ausubel (1968), entre tantos outros.

O que podemos concluir a partir de todas essas teorias, é que, em geral, relacionam-se de alguma forma com o processo de construção do conhecimento, e acabam despertando o interesse para muitas outras pesquisas, como é o caso do estudo em andamento ${ }^{1}$ da autora deste artigo, que contribuiu para a idealização da proposta deste trabalho.

As pesquisas em Educação Matemática (YOKOYAMA, 2012) relacionadas aos anos iniciais do ensino fundamental e associadas aos estudantes com Síndrome de Down (SD), ou com qualquer outro atraso cognitivo, são muito escassas. Não obstante, quando tratamos de recursos tecnológicos (D’AMBROSIO, 1999), é possível afirmar também que existe certa dificuldade proveniente ao estudo de conteúdos ligados à Matemática.

Décadas sobrepõem à essas considerações de modo que, a tecnologia emerge cada vez mais na vida de todos os indivíduos. Nesse patamar, a escola passa a aderir às novas tecnologias, e, sobretudo, quando nos referimos à aprendizagem de conteúdo, especialmente os matemáticos, se faz necessário o estabelecimento de conexões entre as áreas do conhecimento com as situações do cotidiano, oferecendo maior atenção às dificuldades enfrentadas pelos estudantes com algum tipo de prejuízo, seja cognitivo, físico ou sensorial.

Em específico, este estudo se reporta ao conjunto de prejuízos decorrentes da condição estabelecida pela SD relacionados ao processo de construção do conceito de número. A primeira dificuldade enfrentada por estudantes com deficiência intelectual é a quantificação; estudos revelam que o processo de contagem por parte destes estudantes é de difícil compreensão, e o ponta pé inicial para dar autonomia à eles é justamente, fazer com que entendam o conceito de número.

Para tanto, a proposta central deste estudo está associada aos sete processos mentais básicos propostos por Lorenzato (2009) para construir o conceito de número: correspondência; comparação; classificação; sequenciação; seriação; inclusão e conservação.

Em face desta proposta e na intenção de implementar uma possibilidade de aprendizagem em circunstância lúdica ao estudante com SD, foi proposto a construção de um Objeto de Aprendizagem (OA), como recurso pedagógico para auxiliar o professor no processo de ensino e aprendizagem matemática desses estudantes.

Os AO são recursos didáticos na forma de arquivos digitais, imagens, vídeos, referências a sites ou outros materiais que possam ser usados como suporte para as aulas ministradas (SILVA; CAFÉ; CATAPAN; 2010).

Em suma, o uso de OA possibilita o desenvolvimento do raciocínio lógico e estimula a autonomia (JUCÁ, 2006).

Justifica-se que no campo cognitivo, todo aprendizado deve partir do concreto, necessitando de instruções visuais, entre outras, para consolidar o conhecimento. Portanto, o objetivo deste trabalho é descrever a construção metodológica do protótipo de um OA que estimule os sete processos mentais básicos para a construção do conceito de número.

\footnotetext{
${ }^{1}$ Refere-se a pesquisa de mestrado, que vem sendo no âmbito do Programa de Pós-Graduação em Educação da Faculdade de Ciências e Tecnologia - Universidade Estadual Paulista (FCT/UNESP) e inserida na linha de pesquisa "Processos Formativos, Ensino e Aprendizagem". O objetivo da pesquisa consiste em identificar recursos pedagógicos adaptados ou não e estratégias de ensino utilizadas em aulas de Matemática para a construção do conceito de número a estudantes com Síndrome de Down, nas séries iniciais do Ensino Fundamental.
} 


\section{METODOLOGIA}

Este estudo de cunho metodológico pode ser considerado descritivo-analítico e, visa desenvolver um OA que atenda duas condições: 1- os parâmetros para a construção de materiais educacionais digitais baseados no Design Pedagógico definidos por Behar; Torrezzan; Ruckert (2008) e, 2 - o trabalho com os sete processos estabelecidos por Lorenzato (2009) para a construção do conceito de número.

A construção deste estudo é baseada na metodologia de Amante e Morgado (2001), que apresenta quatro etapas: 1. Concepção do projeto; 2. Planificação; 3. Implementação; 4. Avaliação.

Porém, vale destacar que neste estudo, foram realizadas apenas as três primeiras etapas.

Os conteúdos foram selecionados com base nas atividades para sala de aula que Sérgio Lorenzato descreveu na obra - Coleção Formação de Professores: Educação Infantil e Percepção Matemática, que tem como objetivo dar embasamento conceitual aos professores da sala comum seguido de sugestões de atividades para serem desenvolvidas com as crianças, conforme descritos no Quadro 1 - Atividades escolhidas para serem trabalhadas no OA, a partir dos parâmetros apresentados no Quadro 2 - Parâmetros para a construção de materiais educacionais digitais baseados no Design Pedagógico. Ressalta-se que o estudo teve aprovação Ética do Comitê de Ética em Pesquisa sob o CAAE: 83384418.1.0000.5402. 
Quadro 1. Atividades escolhidas para serem trabalhadas no OA

\begin{tabular}{|c|}
\hline Atividade escolhida/Descrição \\
\hline $\begin{array}{l}\text { Correspondência: Cinco cartelas, cada uma com o } \\
\text { desenho diferente de um menino; outras cinco } \\
\text { cartelas, cada uma com o desenho de um mesmo } \\
\text { cachorro. }\end{array}$ \\
\hline $\begin{array}{l}\text { Comparação: Cinco cartelas, cada uma com um só } \\
\text { desenho variando de tamanho; por exemplo: uma } \\
\text { casa, mas cada casa de tamanho diferente das outras } \\
\text { quatro. Outro conjunto de cinco cartelas idêntico a } \\
\text { esse. }\end{array}$ \\
\hline
\end{tabular}

Classificação: Conjunto de cartelas contendo diferentes frutas, bichos, brinquedos, roupas, etc; cada cartela deve ter um só desenho.

Atividade a ser realizada

Pedir à criança que escolha um cachorro para cada menino e que dê o nome a ambos, verificando se ela escolhe nomes diferentes, fazendo a correspondência.

A criança deve comparar os dez desenhos e corresponder os que tiverem os mesmos tamanhos, formando partes.

Sequenciação: Seis objetos de diferentes tamanhos e formas, tais como: botões, sementes, parafusos, porcas, conchas e pedras.

Seriação: Blocos lógicos

Apresentar todas as cartelas de uma vez, pedindo às crianças que separem por algum critério; depois pedir a elas que expliquem quais foram os critérios usados, tais como: número de pernas, cor, ter casca, etc.

A criança deve colocar o objeto em fila e explicar o porquê. É interessante observar como a criança discute a formação da sequência e se já aparece algum critério de ordem.

Apresentar o começo de uma série com duas, três ou quatro peças diferentes (por exemplo, triângulo, círculo e quadrado) e pedir à criança que continue a série, de modo que a ordem das peças se repita. A seriação pode ser feita só de peças com a mesma cor ou com o mesmo tamanho.

Inclusão: Conjunto de sólidos (caixas vazias ou fundos de garrafas plásticas) de tamanhos diferentes, tal que se encaixem uns nos outros.

Conservação de quantidade: Dez botões, sendo cinco grandes e cinco pequenos.

Dar todos os sólidos para a criança e pedir que arranhem todos eles, colocando os menores dentro dos maiores.

Distribuir cinco botões grandes para uma ou mais crianças (grupo A) e, para outras crianças (grupo B), os cinco pequenos; essa distribuição deve ser feita dando um botão por vez e alternadamente aos grupos, para que todos possam perceber que ambos receberam quantidades iguais. A criança deve arrumar os cinco botões grandes da maneira que desejar, em seguida, deve arrumar os cinco botões pequenos, da mesma forma que os grandes foram arrumados. Então o professor propõe a questão: "Qual arrumação tem mais botões, ou as duas têm a mesma quantidade?"

Fonte: Adaptado de Lorenzato (2009). 
Quadro 2 - Parâmetros para a construção de materiais educacionais digitais baseados no Design Pedagógico

\begin{tabular}{|c|c|c|c|c|}
\hline Parâmetros & \multicolumn{4}{|c|}{ Aplicações } \\
\hline Imagem & \multicolumn{2}{|c|}{$\begin{array}{l}\text { Com relação ao conteúdo: Aplicá-las de } \\
\text { modo a apoiar práticas pedagógicas e } \\
\text { não simplesmente como forma de } \\
\text { apresentar uma informação, } \\
\text { possibilitando que o usuário seja capaz } \\
\text { de interpretá-las segundo os seus } \\
\text { conceitos previamente construídos sobre } \\
\text { determinado assunto. Utilizar imagens } \\
\text { estáticas (imagens gráficas e vídeos) e } \\
\text { interativas (animações e simulações) de } \\
\text { modo que o usuário possa criar e testar } \\
\text { hipóteses ao longo dos seus estudos. }\end{array}$} & \multicolumn{2}{|c|}{$\begin{array}{l}\text { Com relação às interfaces: Contextualizar as } \\
\text { interfaces na cultura do usuário e/ou no assunto a } \\
\text { ser abordado pelo material. Utilizar ícones que se } \\
\text { relacionem com os signos do usuário e com a } \\
\text { composição gráfica da interface, de modo a } \\
\text { contemplar o conceito de relevância. Alternar a } \\
\text { utilização de ícones de alta e baixa iconicidade, de } \\
\text { modo a apoiar as práticas pedagógicas aplicadas ao } \\
\text { conteúdo. }\end{array}$} \\
\hline $\begin{array}{l}\text { Navegação e } \\
\text { Usabilidade }\end{array}$ & \multicolumn{2}{|c|}{$\begin{array}{l}\text { Escolher um tipo de navegação (linear, } \\
\text { não linear, mista, Breadcrumbs) ou } \\
\text { planejar a alternância entre diferentes } \\
\text { tipos de navegação de modo a possibilitar } \\
\text { ao aluno uma postura autônoma e } \\
\text { investigativa na maior parte do tempo. } \\
\text { - Aplicar uma estrutura navegacional } \\
\text { compatível com o nível de conhecimento } \\
\text { do usuário a respeito da utilização de } \\
\text { ferramentas informáticas. } \\
\text { - Complementar os critérios ao lado com } \\
\text { os seguintes fatores (também de } \\
\text { usabilidade): visibilidade, feedback, } \\
\text { mapeamento e consistência. } \\
\text {-Elaborar estratégias de affordance } \\
\text { (fornecer pistas a respeito do significado } \\
\text { de um determinado elemento de } \\
\text { interface). }\end{array}$} & \multicolumn{2}{|c|}{$\begin{array}{l}\text { Priorizar a contemplação dos seguintes critérios de } \\
\text { usabilidade: condução, carga de trabalho, controle } \\
\text { explícito, adaptabilidade, gestão de erros, } \\
\text { consistência, expressividade e compatibilidade. }\end{array}$} \\
\hline \multirow[b]{3}{*}{$\begin{array}{c}\text { Interação e } \\
\text { Interatividade }\end{array}$} & \multicolumn{4}{|c|}{ Escolher um tipo de modelo conceitual: } \\
\hline & Baseados em atividades & \multicolumn{2}{|c|}{ Baseado em objetos } & Mistos \\
\hline & $\begin{array}{l}\text { Instrução, conversação, } \\
\text { manipulação e navegação, } \\
\text { exploração e pesquisa. }\end{array}$ & \multicolumn{2}{|c|}{$\begin{array}{l}\text { Interfaces remetem a } \\
\text { objetos comuns de um } \\
\text { certo cotidiano, ex.: } \\
\text { adoção de uma metáfora } \\
\text { de interface ou ícones de } \\
\text { alto grau de iconicidade. }\end{array}$} & $\begin{array}{l}\text { Integrar os modelos conceituais } \\
\text { baseados em atividades aos baseados } \\
\text { em objetos. }\end{array}$ \\
\hline & \multirow[b]{2}{*}{$\begin{array}{l}\text { Planejar o conteúdo de } \\
\text { modo a possibilitar um } \\
\text { diálogo entre o usuário e a } \\
\text { teoria abordada e não a } \\
\text { simples comunicação de } \\
\text { conceitos - comunicação } \\
\text { didática. }\end{array}$} & \multicolumn{3}{|c|}{ Aplicar as seguintes relações (sujeito-conteúdo): } \\
\hline $\begin{array}{l}\text { Organização } \\
\text { do conteúdo }\end{array}$ & & $\begin{array}{l}\text { Relação de } \\
\text { propiciar u } \\
\text { entre o sis } \\
\text { usuário de } \\
\text { aluno sentir- } \\
\text { pelos desaf } \\
\text { desestimulad }\end{array}$ & 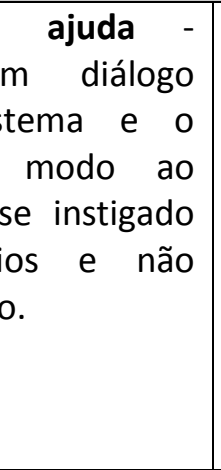 & $\begin{array}{l}\text { Relativização do discurso - possibilitar } \\
\text { que o usuário atue criticamente sobre } \\
\text { o conteúdo como uma das formas de } \\
\text { construção do seu conhecimento. } \\
\text { Desse modo torna-se interessante a } \\
\text { disponibilização de animações e } \\
\text { simulações interativas em que o } \\
\text { usuário possa } \\
\text { elaborar/testar/reconstruir hipóteses } \\
\text { referentes ao conteúdo estudado. }\end{array}$ \\
\hline
\end{tabular}

Fonte: BEHAR; TORREZZAN; RÜCKERT (2008, p.6). 


\section{RESULTADOS}

\subsection{A construção do protótipo do Objeto de Aprendizagem}

De acordo com Ferlin (2009), um OA é dividido em duas áreas: Pedagógica, relacionadas com a concepção de objetos que facilitem o trabalho do professor e o aluno visando a aquisição do conhecimento; e de Técnicas, relacionadas as dimensões de padronização, classificação, e pela programação do software.

As etapas realizadas para a construção do OA estão descritas na Imagem 1 - Processo de Construção do OA. Neste processo, destacamos fundamental a elaboração do storyboard ${ }^{2}$, pois apresenta a descrição de todos os elementos contidos no $\mathrm{OA}$, bem como a apresentação geral do objetivo do jogo em si, destacando minuciosamente a sequência de cada tela, bem como falas do narrador e textos de feedback, elementos que conduzirão os estudantes com SD na realização de cada atividade.

Imagem 1. Processo de construção do AO

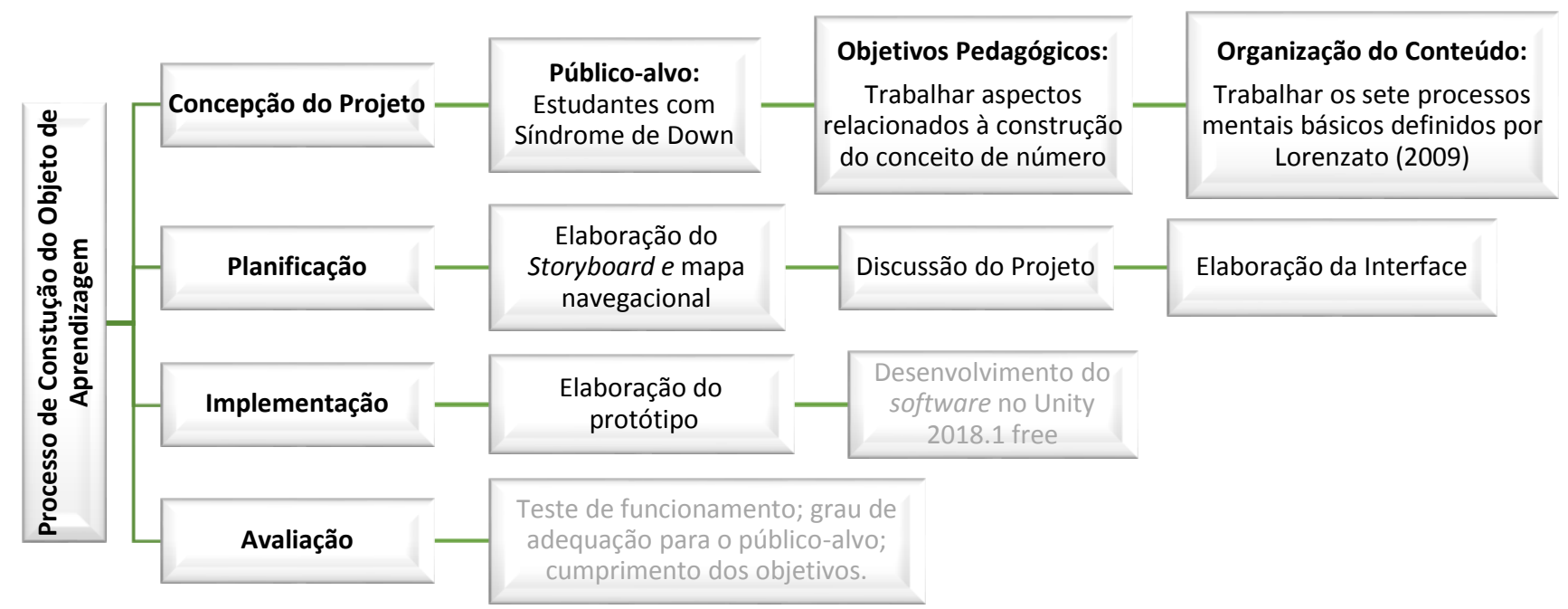

Fonte: Autores (2018).

\footnotetext{
${ }^{2}$ Storyboard é basicamente um roteiro que contém, textos e desenhos em sequência cronológica, como um guia visual, mostrando as cenas e ações de uma obra audiovisual. O storyboard serve para facilitar a comunicação entre os membros da equipe multidisciplinar e produção do recurso, neste caso o OA.
} 
Foram elaboradas sete atividades, descritas como níveis, uma para cada processo mental, conforme descrito anteriormente no Quadro 1 - Atividades escolhidas para serem trabalhadas no OA. Ressalta-se que para a construção do OA adaptamos algumas atividades, visto que nos exemplos de Lorenzato (2009), os materiais utilizados são concretos, e no OA pensamos nas possibilidades que a tecnologia nos oferece, buscando contextualizar e aproximar as atividades para a realidade dos estudantes.

\subsection{Planejamento das Interfaces Gráficas}

Quadro 3. Interface gráfica e objetivo das telas do OA

\begin{tabular}{|l|l|l|l|}
\hline \multicolumn{2}{|c|}{ Interface gráfica/ Objetivo } & \multicolumn{2}{c|}{ Interface gráfica/ Objetivo } \\
\hline & $\begin{array}{l}\text { Tela de cadastro: o } \\
\text { professor deverá inserir } \\
\text { nome e foto de cada um. O } \\
\text { objetivo desta tela é criar } \\
\text { um banco de dados para } \\
\text { cada usuário. }\end{array}$ & $\begin{array}{l}\text { Nível } 01 \text { Correspondência: } \\
\text { corresponder elementos } \\
\text { iguais a lementos } \\
\text { diferentes. }\end{array}$ \\
\hline $\begin{array}{l}\text { Nível 02 - Comparação: } \\
\text { desenvolver a percepção de } \\
\text { tamanhos semelhantes, por } \\
\text { estimativa. }\end{array}$ & $\begin{array}{l}\text { Nível } \mathbf{0 3 ~ - ~ C l a s s i f i c a c ̧ a ̃ o : ~} \\
\text { classificar as imagens com } \\
\text { apenas um atributo, seja ele, } \\
\text { cor, número de pernas, entre } \\
\text { outros. }\end{array}$ \\
\hline
\end{tabular}

\begin{tabular}{|l|l|l|l|}
\hline $\begin{array}{l}\text { Nível } \mathbf{0 4} \text { - Sequenciação: } \\
\text { fazer uma sequência com } \\
\text { algum critério de ordem. É } \\
\text { importante que o professor } \\
\text { pergunte ao aluno qual o } \\
\text { critério ele estabeleceu para } \\
\text { fazer a sequência. }\end{array}$ & $\begin{array}{l}\text { Nível } 05 \text { Seriação: por em } \\
\text { sequência considerando um } \\
\text { só atributo. }\end{array}$ \\
$\begin{array}{l}\text { Nível } 06 \text { - Inclusão: Reforçar } \\
\text { as noções de incluir, conter, } \\
\text { caber, estar dentro, } \\
\text { pertencer. }\end{array}$ & $\begin{array}{l}\text { Nível } \mathbf{0 7} \text { Conservação: } \\
\text { Facilitar a percepção da } \\
\text { conservação de quantidade, } \\
\text { variando tamanho e forma. }\end{array}$ \\
\hline
\end{tabular}

Fonte: Autore (2018).

Como forma de análise dos dados, será realizada a etapa Avaliação após a construção do software. Ao realizar os testes busca-se evidenciar a qualidade do trabalho produzido, verificando erros antes da disponibilização do OA para os estudantes. Estes testes serão voltados à funcionalidade, acessibilidade, confiabilidade técnica e pedagógica, usabilidade, precisão e eficiência.

\section{CONCLUSÃO}

Em síntese, este artigo se propôs a descrever a elaboração do protótipo de um OA baseado nos sete processos de Lorenzato (2009) para a construção do conceito de número, e o planejamento das interfaces gráficas do OA, para que tenha maior aderência de acordo com as necessidades da criança com SD. Os OA facilitam e favorecem a aprendizagem do estudante com $\mathrm{SD}$, por ter um caráter lúdico e motivador. 
Para projeções futuras, considera-se importante a construção do software, bem como a avaliação do mesmo perante testes de funcionalidade, qualidade e aplicabilidade.

\section{REFERÊNCIAS}

AMANTE, L.; MORGADO, L. Metodologia de Concepção e Desenvolvimento de aplicações Educativas: O Caso dos Materiais Hipermídia. In: Discursos, Universidade Aberta, v.3, n. especial, p. 125-138, 2001.

AUSUBEL, D.P. Educational psychology: a cognitive view. New York: Holt, Rinehart and Winston, 1968.

BEHAR, P. A.; TORREZZAN, C. A. W.; RÜCKERT, A. B. PEDESIGN: a construção de um objeto de aprendizagem baseado no design pedagógico. Revistas Novas tecnologias, v.6, n. 2. Dez/2008. D’AMBROSIO, U. A história da matemática: questões historiográficas e políticas e reflexos na Educação Matemática. In: BICUDO, M. A. V.(org.). Pesquisa em Educação Matemática: concepções e perspectivas. São Paulo: UNESP, 1999. p. 97-115.

DEWEY, J. E. E. In: DEWEY, J. The Early Works, 1882-1898. 2. ed. Sourthern Illinois: University Press, 2008. v.5. p. 1895-1898.

FERLIN, J. Repositório de Objetos de Aprendizagem para a área de Informática, 2009, f., Trabalho de Conclusão de Curso (Graduação em...) - Universidade Estadual de Santa Catarina (UDESC), 2009.

JUCÁ, S. C. S. A Relevância dos Softwares Educativos na Educação Profissional. In: Revista Ciências e Cognição, v.8, p. 22-28, 2006.

KAMII, C. A criança e o número: implicações da teoria de Piaget para atuação junto a escolares de 4 a 6 anos. Campinas: Papirus, 1990.

LORENZATO, S. Para aprender Matemática. 2. ed. rev. Campinas: Autores Associados, 2008a. Coleção Formação de Professores.

LORENZATO, S. Que matemática ensinar no primeiro dos nove anos do ensino fundamental, In: CONGRESSO DE LEITURA DO BRASIL, 17., 2009, Campinas. Anais... Campinas: UNICAMP, 2009.

SILVA, L. E; CAFÉ, L; CATAPAN, A. H. Os objetos educacionais, os metadados e os repositórios na sociedade da informação. Ciência da informação. Brasilia, v. 39 n. 3, p. 93-104, set./dez. 2010.

VERGNAUD, G. Teoria dos campos conceituais. In: SEMINÁRIO INTERNACIONAL DE EDUCAÇÃO MATEMÁTICA, 1, 1993, Rio de Janeiro. Anais... Rio de Janeiro: SBEM, 1993. p. 1-26. 